\title{
Cost-effectiveness of early intervention in first-episode psychosis: economic evaluation of a randomised controlled trial (the OPUS study)
}

Lene Halling Hastrup, Christian Kronborg, Mette Bertelsen, Pia Jeppesen, Per Jorgensen, Lone Petersen, Anne Thorup, Erik Simonsen and Merete Nordentoft

\section{Background}

Information about the cost-effectiveness of early intervention programmes for first-episode psychosis is limited.

\begin{abstract}
Aims
To evaluate the cost-effectiveness of an intensive earlyintervention programme (called OPUS) (trial registration NCT00157313) consisting of enriched assertive community treatment, psychoeducational family treatment and social skills training for individuals with first-episode psychosis compared with standard treatment.
\end{abstract}

\section{Method}

An incremental cost-effectiveness analysis of a randomised controlled trial, adopting a public sector perspective was undertaken.

\section{Results}

The mean total costs of OPUS over 5 years ( $€ 123683$, s.e. $=8970)$ were not significantly different from that of standard treatment $(€ 148751$, s.e. $=13073)$. At 2-year followup the mean Global Assessment of Functioning (GAF) score in the OPUS group $(55.16$, s.d. $=15.15)$ was significantly higher than in standard treatment group $(51.13$, s.d. $=15.92)$ However, the mean GAF did not differ significantly between the groups at 5-year follow-up (55.35 (s.d. $=18.28)$ and 54.16 (s.d. = 18.41), respectively). Cost-effectiveness planes based on non-parametric bootstrapping showed that OPUS was less costly and more effective in $70 \%$ of the replications. For a willingness-to-pay up to $€ 50000$ the probability that OPUS was cost-effective was more than $80 \%$.

\section{Conclusions}

The incremental cost-effectiveness analysis showed that there was a high probability of OPUS being cost-effective compared with standard treatment.

\section{Declaration of interest}

None.
Schizophrenia is a severe and chronic illness. ${ }^{1}$ The first psychotic breakdown is usually seen in adolescence or early adulthood. The illness has a serious impact on the young person's life through cognitive-perceptual, emotional and behavioural dysfunction that strongly interfere with their social life and work. ${ }^{2}$ Also, many people with schizophrenia struggle with substance misuse and depression, and the disorder is often associated with high rates of suicide attempts, violence and criminal problems. The importance of focusing on first-episode psychosis arises because delayed detection and treatment of the illness predicts a poor outcome. ${ }^{3-5}$ Furthermore, longer periods of untreated psychosis are associated with poorer outcomes. ${ }^{5}$ Therefore, it is important to identify opportunities for prevention and treatment of the illness. Early-intervention psychiatric services in psychosis seek to help people who are in the early stages of their illness and are experiencing their first episode of psychosis. The treatment combines enriched assertive community treatment with psychoeducation and family intervention. Evidence for the effectiveness of early-intervention services targeting people in the earlier phases of a diagnosed psychosis has been demonstrated in various studies. ${ }^{6}$ In particular, randomised controlled trials (RCTs) such as the OPUS study in Denmark and the LEO (Lambeth Early Onset) study in the UK have demonstrated a better outcome in the short term (less than 2 years) for specialised early-intervention programmes over standard treatment on a broad range of outcomes including psychotic and negative symptoms, vocational outcome, social functioning, reduced number of bed days in psychiatric in-patient units and improved treatment adherence. ${ }^{7-9}$ In spite of the rapid development of early-intervention services during the past decade, the evidence base for the health economics of these services is still limited with only a few published studies. ${ }^{10-14}$ The current evidence on cost-effectiveness is based on studies with historical controls or studies without enough power in terms of patient numbers to answer health economic questions definitively. ${ }^{10,15}$ In particular, the question of whether early- intervention services are cost-effective in the long term has not been addressed. ${ }^{15}$ In this study, we aimed to analyse the cost- effectiveness of an intensive early-intervention programme, using data from the largest trial to date, and comparing it with standard community treatment.

\section{Method}

\section{Setting and participants}

Our cost-effectiveness analysis was based on a single-blind, randomised controlled clinical study comparing an intensive early-intervention programme (called OPUS) with standard treatment (in community mental health centres) in Copenhagen and Aarhus in Denmark. A total of 547 patients in contact with in-patient or out-patient mental health services for the first time were consecutively included in the study from January 1998 to December 2000. The 5-year follow-up rates were 56\% (151 patients) in OPUS and 57\% (150 patients) in the control group. In order to assess the influence of missing data on the 5-year patient results, outcome measures were subject to further analysis and no statistically significant differences were found between patients attending the 5-year follow-up and those who had dropped out. ${ }^{7}$

At the time of inclusion, patients were between 18 and 45 years of age and had a clinical diagnosis within the schizophrenia spectrum (ICD-10 codes in the F2 category ${ }^{16}$ ). None of the 
patients had previously received antipsychotic medication for more than 12 continuous weeks.

Full details of the study design and intervention are described in the papers presenting the clinical results of the OPUS trial. $^{7,9,17,18}$

\section{OPUS}

The intensive early-intervention programme consisted of an enriched assertive community treatment inspired by Stein \& Test, ${ }^{19}$ psychoeducational family treatment modelled on McFarlane et al's manual for multifamily groups, ${ }^{20,21}$ social skills training, ${ }^{22}$ and low-dose medication. Each patient was offered treatment for 2 years (followed by transition to standard treatment) by a multidisciplinary team providing the integrated treatment. The case-load was planned as 1 staff member for each 10 patients. A primary team member was designated for each patient and they were responsible for maintaining contact and coordinating treatment within the team and across different treatment and support facilities. Patients were visited in their homes or other places in their community or at their primary team member's office according to the patient's preference. A crisis plan was developed for each patient.

\section{Standard treatment}

Standard treatment consisted of the standard routine care offered by the mental health services in Copenhagen and Aarhus at that time and patients with first-episode psychosis were mixed in with people with a spectrum of other mental disorders. Appointments were usually held at the local community mental health centres and the patients were in contact with a physician, a psychiatric nurse and, in some cases, a social worker. Case-load varied between 20 and 30 patients per member of staff. Administration of antipsychotic medication was based on the same principles as in the OPUS treatment.

\section{Type of evaluation, perspective and length of study}

The objective of the economic analysis was to assess the relative cost-effectiveness of OPUS in comparison with standard treatment. The evaluation adopted a public sector perspective when considering the costs associated with early-intervention services (including those for healthcare and the supported housing facility). Cost-effectiveness was assessed by calculating the incremental cost-effectiveness ratio (ICER). ${ }^{23}$ The uncertainty surrounding the estimates of expected costs and expected outcomes was estimated by non-parametric bootstrapping of 2000 replicates of the ICER. The cost-effectiveness acceptability curve (CEAC) was estimated as the probability that the OPUS intervention was cost-effective compared with standard treatment, given observed data, for a range of monetary values that a decision-maker might be willing to pay for a unit increase in health outcome measure. ${ }^{24}$ The study period was 5 years.

\section{Resource use}

We extracted all resource data covering 1998 to 2007 for all patients in the study by their personal identification number registered in the Danish Civil Registration System. Since the patients were consecutively enrolled into the study, we classified the use of services in 1-year intervals from 1 to 5 years after the inclusion date.

Data on psychiatric in-patient and out-patient treatment, and contacts with psychiatric emergency departments were extracted from the Danish Psychiatric Central Register. ${ }^{25}$ Out-patient data were collected by interviewers during the trial. These data were supplemented by out-patient data extracted from the Danish Psychiatric Central Register for the 3 years following the intervention period.

Data on somatic in-patient and out-patient treatment and visits to hospital accident and emergency rooms were extracted from the National Patient Registry, which is a central registry of all discharges of individual patients from publicly owned hospitals including out-patient visits and accident and emergency department visits. ${ }^{26}$ Data on the use of services from general practitioners (GPs) and other medical specialists, dentists, physiotherapists, chiropractors, chiropodists, and psychological counsellors were obtained from the National Health Insurance Service Register, which is a central registry of healthcare services that are reimbursed by National Health Insurance. ${ }^{27}$ Data on all patients' use of prescription drugs were collected from the Register of Medicinal Product Statistics, which is a central registry based on transaction reports from the dispensing pharmacies. ${ }^{28}$ It is administered by the Danish Medicines Agency. We collected information on number of days living in supported houses for patients with mental health problems by combining a database with addresses for all supported housing facilities in Denmark with address information in the Civil Status Register.

\section{Costs}

The resource volumes were combined with unit costs to obtain a cost per person over their time in the study. All costs in the study were calculated in 2009 values of Danish Kroners and converted to Euros $(1 €=7.44 \mathrm{DKK})$ both undiscounted and in present values by discounting costs by the annual rate of $3 \%$. (In health economic evaluations, future costs (and sometimes health gains) are commonly weighted in relation to the time at which they occur. Future costs receiving less weight than present ones.)

Since diagnosis-related group (DRG) charges for psychiatric services were not developed in Denmark for this time period, we estimated the cost of bed days in psychiatric hospitals, outpatient contacts and contacts in emergency wards by multiplying the number of bed days and contacts with fixed charges obtained from the Danish National Board of Health. ${ }^{26}$ These charges are based on historical cost data, and were considered to approximate public sector opportunity cost. We used DRG charges as unit costs for somatic hospital treatment. The DRG charges were provided by the Danish National Board of Health and reflect the average cost of treating patients with similar conditions in a Danish hospital. For patients who stayed beyond the number of days covered by the DRG charge, an additional charge per day in excess of the number of days covered was added to the hospital costs in accordance with the Danish DRG charge guidelines. In Denmark, hospital treatment is provided free of charge to the patient. National DRG charges are used for reimbursements between regional healthcare authorities providing hospital treatment if a patient from one region is treated at a hospital in another region. These estimates are based on detailed accounts of resource use per patient group in prior years, and are therefore deemed to represent a good approximation of opportunity costs.

The unit costs of GP services and services from other healthcare providers were based on the prevailing National Health Insurance fee schedules. ${ }^{26}$ We estimated the costs of living in supported housing facilities by multiplying the number of days in supported housing by a mean charge that represents the reimbursement that supported houses receive from local authorities. This charge varies between the different supported houses depending on the size and support facilities. Since we have no information about which of the supported houses in the area 


\begin{tabular}{|c|c|c|c|}
\hline & \multirow[b]{2}{*}{ Base case } & \multicolumn{2}{|c|}{ Values used in sensitivity analysis } \\
\hline & & $\begin{array}{l}\text { Applying lowest } \\
\text { observed unit cost }\end{array}$ & $\begin{array}{l}\text { Applying highest } \\
\text { observed unit cost }\end{array}$ \\
\hline I. OPUS intervention costs per year & 8445 & & \\
\hline Staff costs & 9774 & 8574 & 11173 \\
\hline II. Costs of standard treatment: case-load (staff/patient ratio) of standard treatment & $1: 25$ & $1: 30$ & $1: 20$ \\
\hline Cost of standard treatment per year & 3437 & 2864 & 4229 \\
\hline III. Mean estimated cost per day for supported housing & 321 & 253 & 512 \\
\hline
\end{tabular}

the patients had been assigned to, we used information from the National Board of Social Services on charges of all housing facilities to estimate a mean charge regarding reimbursement that the housing facilities in the Copenhagen and Aarhus area receive per bed day.

In relation to cost assessment, the number of staff per patient treated was the most important difference between OPUS and standard treatment. Whereas OPUS had a case-load of 10 patients per member of staff, the case-load for standard treatment varied between 20 and 30 patients per member of staff. Hence we based our calculation on this information and used a top-down approach to assess the intervention costs and costs of standard treatment.

We estimated the staff cost of one OPUS team as being made up of one full-time: psychiatrist, psychiatric nurse, psychologist, social worker, occupational therapist and secretary. Further, one full-time labour market/educational guide was recruited to the Copenhagen teams in 2000. We added variable costs for transportation, medical drugs and educational and supervision costs of the staff. Fixed costs/operating costs (i.e. rent, electricity, heating and water) were also included. The costs relating to standard treatment were assessed by estimating staff costs of a physician, a psychiatric nurse and a social worker and we included variable and fixed costs.

For the standard treatment we assumed a case-load of 1 member of staff to 25 patients. In sensitivity analysis, we analysed the ways in which the staff costs and charges for supported living facilities affected the overall costs. Further, we examined how different case-loads for standard treatment (1:20 and 1:30) affected the overall costs. Table 1 shows the unit costs that were used for estimation of costs.

\section{Outcome}

The primary clinical outcome measure was assessment of overall mental health functioning using the Global Assessment of Functioning (GAF) scale. ${ }^{7}$ The GAF is on a $1-100$ scale divided into ten deciles, each of which provides a description of functioning level. A higher score on GAF denotes a better functional level.

\section{Statistical analysis}

Data were analysed on an intention-to-treat basis. No statistically significant differences were found between treatment groups at baseline in terms of either clinical or sociodemographic characteristics. ${ }^{18}$ Patients who died during the observation period (7 in OPUS, 10 in the control group) were excluded from the analysis. Resource use and cost data for complete cases were analysed in accordance with the 5-year follow-up of the clinical trial. Mean differences between both groups are presented with their 95\% confidence intervals. We estimated confidence intervals by non-parametric bootstrapping as a check of the robustness of the standard parametric $t$-test. Since we found only minor differences, which did not influence the results, the non-parametric tests are not reported here. Cost-effectiveness was evaluated by relating differences in total costs per patient to differences in effectiveness (GAF) across treatment groups. The ICER was calculated as the difference in mean cost divided by the difference in mean GAF scores at 5 -year follow-up. ${ }^{23}$ To reflect the uncertainty in the estimates of mean costs and effects, a scatter plot of the 2000 bootstrapped incremental costs and effect is presented on the incremental cost-effectiveness plane shown in Fig. 1. The CEAC was generated by calculating the proportion of 2000 iterations where the incremental cost-effectiveness ratio was below a given threshold. ${ }^{29}$ All data were analysed using Stata SE 11.0 for Windows at Statistics Denmark's server via remote access.

\section{Results}

\section{Outcome data}

The primary outcome measure (GAF) demonstrated an effect for OPUS at the 2-year follow-up. The mean GAF score in the OPUS group was statistically significantly higher than in the control group (difference 3.12, 95\% CI 0.37-5.88). ${ }^{7}$ The mean GAF scores at 2- and 5-year follow-up are presented in Table 2. A statistically significant difference between OPUS and the control group was not present at the 5-year follow-up (difference 1.19, 95\% CI -2.65 to 5.34$){ }^{7}$

\section{Resource use}

The mean number of psychiatric bed days over 5 years was 46 days lower in the OPUS group than in the control group (95\% CI -89.38 to -3.87 ) (Table 3 ). As expected, the intervention increased the number of psychiatric out-patient visits compared with standard treatment. Hence, the mean number of psychiatric out-patient visits was 53 days higher in the OPUS than the control group (95\% CI 41.43-64.93). The number of days in supported housing facilities was 58 days lower in the OPUS group over 5 years $(95 \%$ CI 0.25 to -115.55$)$. No other differences in resource use between the two groups were identified.

\section{Costs}

Over 5 years, the mean total costs of OPUS were $€ 123683$ $($ s.e. $=8970)$, whereas the mean total costs of standard treatment

\begin{tabular}{|c|c|c|}
\hline \multirow[b]{2}{*}{ Follow-up } & \multicolumn{2}{|c|}{ Treatment group, mean (s.d.) } \\
\hline & OPUS & Control \\
\hline 2 years & $55.16(15.15)$ & 51.13 (15.92) \\
\hline 5 years & 55.35 (18.28) & $54.16(18.41)$ \\
\hline
\end{tabular}




\begin{tabular}{|c|c|c|c|}
\hline \multirow[b]{2}{*}{ Resources used, $n$} & \multicolumn{2}{|c|}{ Mean (s.e.) } & \multirow[b]{2}{*}{ Mean difference $(95 \% \mathrm{Cl})$} \\
\hline & OPUS group & Control group & \\
\hline Bed days in psychiatric hospital & $147.25(13.67)$ & $193.88(16.97)$ & $-46.63(-89.38$ to -3.87$)$ \\
\hline Psychiatric out-patient visits & $109.65(4.48)$ & $56.46(3.96)$ & 53.18 (41.43 to 64.93) \\
\hline Visits to psychiatric emergency rooms & $3.27(0.39)$ & $3.82(0.54)$ & $-0.55(-1.86$ to 0.76$)$ \\
\hline Bed days in somatic hospital & $3.57(0.88)$ & $3.71(0.89)$ & $-0.14(-2.59$ to 2.32$)$ \\
\hline Out-patient visits & $2.07(0.32)$ & $1.57(0.19)$ & $0.50(-0.24$ to 1.23$)$ \\
\hline Visits to emergency rooms & $2.16(0.28)$ & $2.20(0.32)$ & $-0.04(-0.88$ to 0.79$)$ \\
\hline General practitioner consultations & $16.79(1.01)$ & $17.43(1.71)$ & $-0.64(-4.53$ to 3.25$)$ \\
\hline Telephone consultations & $19.80(1.33)$ & $19.33(1.53)$ & $0.47(-3.98$ to 4.91$)$ \\
\hline Consultations with psychiatrist & $1.11(0.28)$ & $0.90(0.28)$ & $0.21(-0.58$ to 0.98$)$ \\
\hline Consultations with psychologist & $0.12(0.07)$ & $0.03(0.03)$ & $0.09(-0.06$ to 0.25$)$ \\
\hline Packs of prescription drugs & $82.22(9.41)$ & $83.28(11.45)$ & $-1.06(-30.14$ to 28.03$)$ \\
\hline Days in supported housing facilities (complete cases) & $84.27(17.37)$ & $141.92(24.07)$ & $-57.65(-115.55$ to 0.25$)$ \\
\hline
\end{tabular}

were $€ 148651($ s.e. $=13073)$ (Table 4). Table 5 shows that the total costs of OPUS were statistically significantly lower in year 4 (difference $7407,95 \%$ CI -14456 to -359 ). The psychiatric hospital costs were statistically significantly lower in OPUS in the first and third year after inclusion, whereas the psychiatric out-patient costs were higher in OPUS during the first 3 years. There is no difference in costs of supported housing facilities during the first 3 years, but at 4 and 5 years after inclusion, the costs were lower in OPUS. We found no differences in costs for somatic hospital treatment, medical specialists or prescription drugs over the 5 years.

One-way sensitivity analyses were undertaken to explore the impact on the base-case results of changing underlying assumptions of the costing analyses (e.g. changing the staff costs, the case-load for standard treatment, the unit price of supported housing facilities). The results demonstrated that the base-case analysis was robust to different assumptions in the costing analysis. The differences in costs remained statistically insignificant.

\section{Cost-effectiveness analysis}

In total, $70 \%$ of the points are in the south-eastern quadrant of the cost-effectiveness plane, which represents the position where the intervention is more effective and less costly than the standard treatment (Fig. 1). Figure 1 also shows the point estimate of the ICER.

Figure 2 demonstrates that the probability of the treatment being less costly and more effective than standard treatment is $95.3 \%$, which represents the probability of it being cost-effective when the decision maker is unwilling to incur additional costs for an extra point increase in the GAF. If the decision maker is willing to pay $€ 2000$ per point increase in the GAF, the probability rises to $96.5 \%$.

\section{Discussion}

The economic evaluation revealed that there was no statistically significant difference in total mean costs between OPUS and standard treatment over 5 years. The difference in health outcome had disappeared at the 5-year follow-up. The incremental costeffectiveness analysis showed that there was a high probability of OPUS being cost-effective compared with standard treatment.

To our knowledge, no other studies have analysed the cost-effectiveness of early intervention in psychosis based on an RCT in such a large patient group during an extended 5-year follow-up period. In the LEO study ${ }^{12}$ in south London, 114 patients with newly diagnosed psychosis were randomised to an intervention by a multidisciplinary assertive outreach team. The follow-up period in this study was 18 months. The intervention was comparable with the OPUS intervention and consisted of cognitive-behavioural therapy, family therapy, vocational rehabilitation and low-dose medication regimes. Standard treatment was provided by a community mental health team with no training in dealing with first-episode psychosis. As in the OPUS evaluation, the overall costs showed no statistically significant differences between the treatment groups. The LEO study found a reduction of one-third of psychiatric in-patient

Table 4 Mean cumulative costs (2009 $€$ ) per person over 5 years (undiscounted unless stated otherwise), complete cases

\begin{tabular}{|c|c|c|c|c|c|}
\hline & $\begin{array}{l}\text { OPUS group } \\
\text { Mean (s.e.) }\end{array}$ & $\begin{array}{l}\text { Control group } \\
\text { Mean (s.e.) }\end{array}$ & $\begin{array}{l}\text { Difference } \\
\text { (s.e.) }\end{array}$ & $95 \% \mathrm{Cl}$ & $P$ \\
\hline Psychiatric hospital & $58502(5437)$ & 77021 (6748) & $-18519(8656)$ & -35523 to -1515 & $<0.032$ \\
\hline Out-patient visits (psychiatric) & 21783 (891) & 11249 (789) & 10534 (1191) & 8195 to 12874 & $<0.000$ \\
\hline Emergency rooms (psychiatric) & $650(79)$ & $760(107)$ & $-110(133)$ & -372 to 151 & $<0.407$ \\
\hline Somatic hospital & $2064(284)$ & 3118 (737) & $-1054(787)$ & -2599 to 492 & $<0.181$ \\
\hline Out-patient and emergency room visits & $889(97)$ & 800 (93) & 89 (134) & -174 to 352 & $<0.507$ \\
\hline Prescription drugs & $5544(465)$ & $5311(465)$ & $233(657)$ & -1058 to 1524 & $<0.723$ \\
\hline Medical specialists & 2607 (367) & $2513(235)$ & $94(437)$ & -766 to 953 & $<0.831$ \\
\hline Supported housing facilities & 26051 (5371) & 43874 (7441) & $-17823(9109)$ & 77 to -35722 & $<0.051$ \\
\hline Total costs ${ }^{a}$ & $123683(8970)$ & $148651(13073)$ & $-24968(15719)$ & 3447 to -58330 & $<0.113$ \\
\hline Total cost, discounted rate $3 \%$ & $111924(8208)$ & 137638 (12049) & $-25714(14453)$ & -54113 to 2685 & $<0.110$ \\
\hline
\end{tabular}



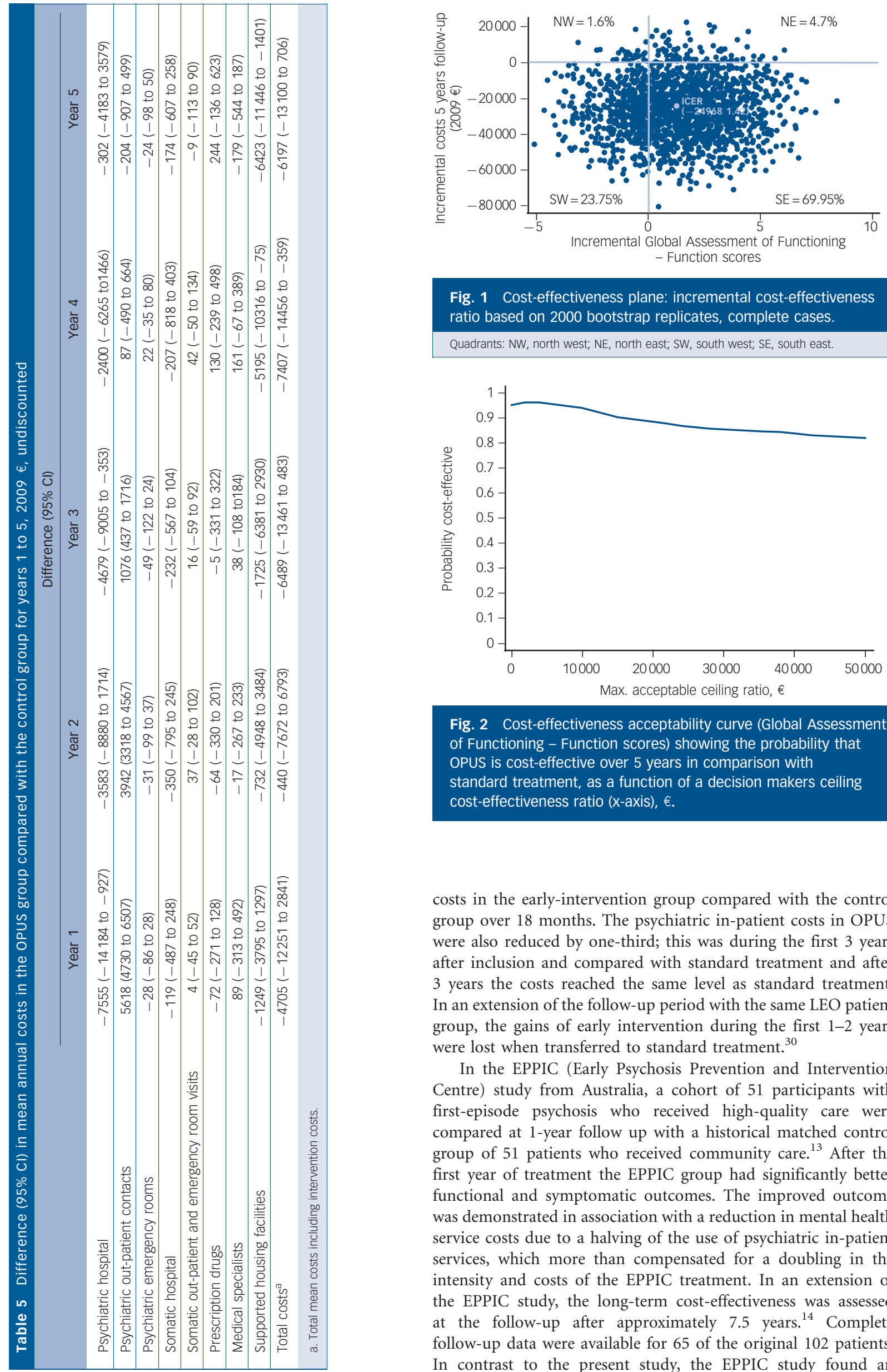

Fig. 1 Cost-effectiveness plane: incremental cost-effectiveness ratio based on 2000 bootstrap replicates, complete cases.

Quadrants: NW, north west; NE, north east; SW, south west; SE, south east.

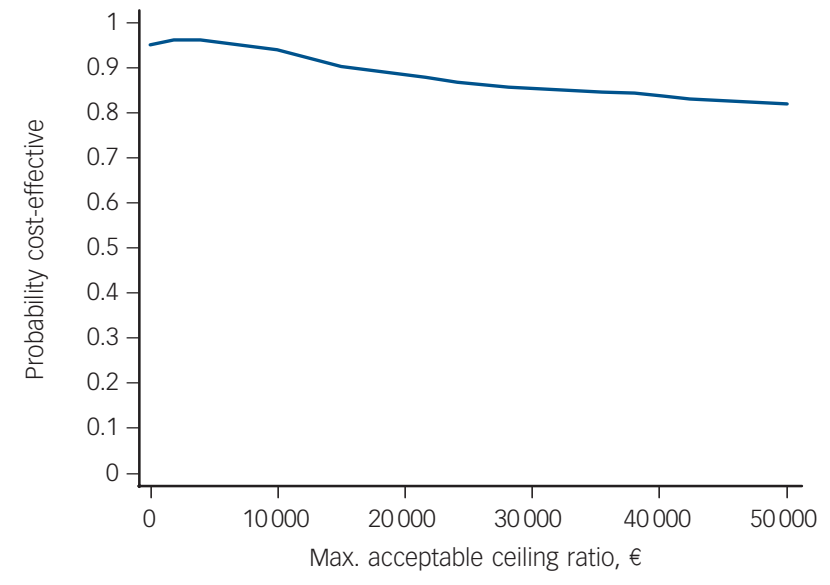

Fig. 2 Cost-effectiveness acceptability curve (Global Assessment of Functioning - Function scores) showing the probability that OPUS is cost-effective over 5 years in comparison with

standard treatment, as a function of a decision makers ceiling cost-effectiveness ratio (x-axis), $€$.

costs in the early-intervention group compared with the control group over 18 months. The psychiatric in-patient costs in OPUS were also reduced by one-third; this was during the first 3 years after inclusion and compared with standard treatment and after 3 years the costs reached the same level as standard treatment. In an extension of the follow-up period with the same LEO patient group, the gains of early intervention during the first $1-2$ years were lost when transferred to standard treatment. ${ }^{30}$

In the EPPIC (Early Psychosis Prevention and Intervention Centre) study from Australia, a cohort of 51 participants with first-episode psychosis who received high-quality care were compared at 1-year follow up with a historical matched control group of 51 patients who received community care. ${ }^{13}$ After the first year of treatment the EPPIC group had significantly better functional and symptomatic outcomes. The improved outcome was demonstrated in association with a reduction in mental health service costs due to a halving of the use of psychiatric in-patient services, which more than compensated for a doubling in the intensity and costs of the EPPIC treatment. In an extension of the EPPIC study, the long-term cost-effectiveness was assessed at the follow-up after approximately 7.5 years. ${ }^{14}$ Complete follow-up data were available for 65 of the original 102 patients. In contrast to the present study, the EPPIC study found an 
advantage both in terms of clinical outcomes and treatment costs, which was maintained beyond the period over which the intervention was provided. The EPPIC patients had better functional outcome (GAF) and lower levels of positive psychotic symptoms (Brief Psychiatric Rating Scale total) than the historical controls at follow-up. Moreover, the mean total costs per EPPIC patient, including mental healthcare and medication costs, were one-third of the costs of the control group. The authors, however, acknowledge the caveats of the study that among others include a small sample size and large attrition $(36 \%)$ of the original cohort of 102 patients. In the Swedish 'Parachute Project', 61 patients with first-episode psychotic were followed over 3 years and compared with two other treatment groups. ${ }^{10}$ The study assessed mental healthcare costs and found that the psychiatric in-patient costs in the first-episode group were one-third of the control group, whereas the out-patient costs were more than four times higher in the first-episode group. The study found no differences in costs 2 and 3 years after inclusion.

\section{Strengths and weaknesses of the study}

A major strength of the present study is the design of the trial, which was conducted as an RCT in a routine psychiatric out-patient setting, with a relatively large patient sample and a long follow-up period. Further, the study relies on data drawn from official Danish registers, which are known to be of high quality and characterised by a high degree of completeness and validity. ${ }^{31-33}$ The limitations include the fact the study did not include indirect costs derived from contacts with the criminal justice system or care provided by informal caregivers, such as family and friends. Informal caregiving is unpaid but may clearly carry an economic cost since the time used for informal caregiving usually could be used for other purposes. ${ }^{34}$ Other studies among patients with schizophrenia have shown that informal caregiving is substantial in this patient group. ${ }^{35}$

In addition, productivity loss was not included in the study, but we calculated the costs of early retirement pension as an indicator of the patients' ability to be a part of the labour force. There appeared to be no difference between the treatment groups in the costs of early retirement pension in the 5 years after inclusion in the study. Likewise, there was no difference in the proportion of patients who had a job or were in education after 5 years (data not shown here). ${ }^{7}$ Since the economic evaluation was not planned from the beginning of the trial, the trial was powered to measure differences in health outcome but a power analysis was not performed for economic evaluation. Another limitation was that a preference-based quality of life measure was not available as a supplement to GAF, as an outcome measure for the economic evaluation.

A top-down approach was used to assess the intervention costs and standard treatment based on staff costs, variable and fixed costs. An average cost per patient treated in the OPUS and standard treatment group respectively was calculated by dividing the costs by the number of patients. Hence, there may be cost differences, which are not captured by the average cost per patient, for example cost differences across types of members of staff or use of individual $v$. group therapy. We believe, however, that the calculated average costs reflect the additional costs of the OPUS intervention due to the higher case-load per patient treated compared with standard treatment.

Unit costs were based on tariffs or charges and therefore do not reflect exact estimates of the true opportunity costs. There may be some variations in the costs of psychiatric in-patient services, which are not accurately reflected in the average unit cost applied. Nevertheless, as most of the services that are included in our analysis are provided by the national healthcare service (i.e. non-profit organisations) the reimbursement rates are likely to be reasonable reflections of the costs of services.

\section{Implications for clinical practice and for policy makers}

Since the difference in effect on the primary outcome measures disappeared during the period of 3 years following transition to standard treatment, it is has been suggested that the 2-year intervention period is too short, at least for some of the patients. ${ }^{7}$ Currently, there is no evidence regarding the optimal duration of specialised early-intervention treatment to prevent relapse $^{7}$ or which specific elements of early intervention need to be offered for an extended period to prevent relapse. Birchwood et al hypothesised that there is a critical period up to 5 years after onset of psychosis, which represents a window of opportunity where the long-term course can be influenced. ${ }^{3}$ An RCT, OPUS-II, is being carried out in Denmark in order to test whether an extension of the specialised intervention up to 5 years will allow the beneficial effects to continue beyond the critical phase, through consolidation of improved social and functional outcomes. ${ }^{2}$ In the light of this evaluation, the costs of a 5-year intervention seem to depend on the intensity of the intervention in terms of out-patient treatment costs and on supported housing facility costs, since the psychiatric in-patient costs reached the same low level in both treatment groups 4 years after the intervention.

\begin{abstract}
Lene Halling Hastrup, PhD, Region Zealand, Psychiatric Research Unit; Christian Kronborg, MSC, PhD, Southern Danish Univeristy, Institute of Business and Economics, Centre for Health Economics Research; Mette Bertelsen, PhD Economics, Centre for Health Economics Research; Mette Bertelsen, PhD,
Pia Jeppesen, PhD, University of Copenhagen, Department of Psychiatry, Bispebjerg Hospital; Per Jorgensen, PhD, Board for Mental and Social Affairs, Central Region of Denmark; Lone Petersen, PhD, Anne Thorup, PhD, University of Copenhagen, Department of Psychiatry, Bispebjerg Hospital; Erik Simonsen, PhD, Region Zealand, Psychiatric Research Unit; Merete Nordentoft PhD, University of Copenhagen, Department of Psychiatry, Bispebjerg Hospital, Denmark

Correspondence: Lene Halling Hastrup, Region Zealand, Psychiatric Research Unit, Toftebakken 9, DK-4000 Roskilde, Denmark. Email: Ihhs@regionsjaelland.dk

First received 21 Mar 2012, final revision 9 Aug 2012, accepted 3 oct 2012
\end{abstract}

\section{References}

1 World Health Organization. Impact of Disorders. World Health Organization, 2001.

2 Melau M, Jeppesen P, Thorup A, Bertelsen M, Petersen L, Gluud C, et al. The effect of five years versus two years of specialised assertive intervention for first episode psychosis - OPUS II: study protocol for a randomized controlled trial. Trials 2011; 12: 72.

3 Birchwood $M$, Todd $P$, Jackson C. Early intervention in psychosis. The critical period hypothesis. Br J Psychiatry 1998; 172 (suppl 33): 53-9.

4 Jeppesen $\mathrm{P}$, Petersen L, Thorup A, Abel MB, Ohlenschlaeger J, Christensen TO, et al. The association between pre-morbid adjustment, duration of untreated psychosis and outcome in first-episode psychosis. Psychol Med 2008; 38: 1157-66.

5 Marshall M, Lewis S, Lockwood A, Drake R, Jones $\mathrm{P}$, Croudace T. Association between duration of untreated psychosis and outcome in cohorts of firstepisode patients: a systematic review. Arch Gen Psychiatry 2005; 62: 975-83.

6 McGorry P, Johanessen JO, Lewis S, Birchwood M, Malla A, Nordentoft M, et al. Early intervention in psychosis: keeping faith with evidence-based health care. Psychol Med 2010; 40: 399-404.

7 Bertelsen $\mathrm{M}$, Jeppesen $\mathrm{P}$, Petersen L, Thorup A, øhlenschlaeger J, le Quach $P$, et al. Five-year follow-up of a randomized multicenter trial of intensive early intervention vs standard treatment for patients with a first episode of psychotic illness: the OPUS trial. Arch Gen Psychiatry 2008; 65: 762-71.

8 Craig TK, Garety P, Power P, Rahaman N, Colbert S, Fornells-Ambrojo M, et al. The Lambeth Early Onset (LEO) team: randomised controlled trial of the effectiveness of specialised care for early psychosis. BMJ 2004; 329: 1067. 
9 Petersen L, Jeppesen P, Thorup A, Abel MB, Ohlenschlaeger J, Christensen TO, et al. A randomised multicentre trial of integrated versus standard treatment for patients with a first episode of psychotic illness. BMJ 2005; 331: 602.

10 Cullberg J, Mattsson M, Levander S, Holmqvist R, Tomsmark L, Elingfors C et al. Treatment costs and clinical outcome for first episode schizophrenia patients: a 3-year follow-up of the Swedish "Parachute Project" and two comparison groups. Acta Psychiatr Scand 2006; 114: 274-81.

11 Goldberg K, Norman R, Hoch JS, Schmitz N, Windell D, Brown N, et al. Impact of a specialized early intervention service for psychotic disorders on patien characteristics, service use, and hospital costs in a defined catchment area. Can J Psychiatry 2006; 51: 895-903.

12 McCrone P, Craig TKJ, Power P, Garety PA. Cost-effectiveness of an early intervention service for people with psychosis. Br J Psychiatry 2010; 196 $377-82$

13 Mihalopoulos C, McGorry PD, Carter RC. Is phase-specific, communityoriented treatment of early psychosis an economically viable method of improving outcome? Acta Psychiatr Scand 1999; 100: 47-55.

14 Mihalopoulos C, Harris M, Henry L, Harrigan S, McGorry P. Is early intervention in psychosis cost-effective over the long term? Schizophr Bull 2009; 35: 909-18.

15 Malla A, Pelosi AJ. Is treating patients with first-episode psychosis cost-effective? Can J Psychiatry 2010; 55: 3-7.

16 World Health Organization. The ICD-10 Classification of Mental and Behavioural Disorders: Clinical Descriptions and Diagnostic Guidelines. WHO, 1992.

17 Jorgensen P, Nordentoft M, Abel MB, Gouliaev G, Jeppesen P, Kassow P. Early detection and assertive community treatment of young psychotics: the Opus Study Rationale and design of the trial. Soc Psychiatry Psychiatr Epidemiol 2000; 35: 283-7.

18 Petersen L, Nordentoft M, Jeppesen P, Øhlenschaeger J, Thorup A Christensen TO, et al. Improving 1-year outcome in first-episode psychosis: OPUS trial. Br J Psychiatry 2005; 187 (suppl 48): s98-103.

19 Stein LI, Test MA. Alternative to mental hospital treatment. I. Conceptua model, treatment program, and clinical evaluation. Arch Gen Psychiatry 1980; 37: 392-7.

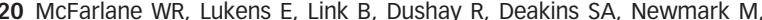
et al. Multiple-family groups and psychoeducation in the treatment of schizophrenia. Arch Gen Psychiatry 1995; 52: 679-87.
21 McFarlane WR. Multifamily Groups in the Treatment of Severe Psychiatric Disorders, Guilford Press, 2002.

22 Liberman RP, Wallace CJ, Blackwell G, Kopelowicz A, Vaccaro JV, Mintz J. Skills training versus psychosocial occupational therapy for persons with persistent schizophrenia. Am J Psychiatry 1998; 155: 1087-91.

23 Glick H, Doshi JA, Sonnad SS, Polsky D. Economic Evaluation in Clinical Trials. New York: Oxford University Press, 2007.

24 Fenwick $E$, Byford $S$. A guide to cost-effectiveness acceptability curves. Br J Psychiatry 2005; 187: 106-8.

25 Mors O, Perto GP, Mortensen PB. The Danish Psychiatric Central Research Register. Scand J Public Health 2011; 39 (suppl 7): 54-7.

26 National Board of Health. Takstsystem 2009 (Hospital charges 2009). Sundhedsstyrelsen, 2011

27 The National Health Insurance Service. Sygesikringens Administrative Vejledning - Takster (The National Health Insurance Service - Charges). Den Offentlige Sygesikring. Danske Regioner (Danish Regions), 2009.

28 Kildemoes HW, Sørensen HT, Hallas J. The Danish National Prescription Register. Scand J Public Health 2011; 39: 38-41.

29 Drummond MF, Sculpher MJ, Torrance GW, O'Brien BJ, Stoddart GL. Methods for the Economic Evaluation of Health Care Programmes (3rd edn). Oxford University Press, 2005

30 Gafoor R, Nitsch D, Mccrone P, Craig TKJ, Garety PA, Power P, et al. Effect of early intervention on 5-year outcome in non-affective psychosis. Br J Psychiatry 2010; 196: 372-6.

31 Andersen TF, Madsen M, Jorgensen J, Mellemkjoer L, Olsen JH. The Danish National Hospital Register. A valuable source of data for modern health sciences. Dan Med Bull 1999; 46: 263-8.

32 Munk-Jorgensen $\mathrm{P}$, Mortensen PB. The Danish Psychiatric Central Register Dan Med Bull 1997; 44: 82-4.

33 Olivarius NF, Hollnagel $H$, Krasnik A, Pedersen PA, Thorsen $H$. The Danish National Health Service Register. A tool for primary health care research. Dan Med Bull 1997; 44: 449-53.

34 Mccrone $P$. Health economic measures in schizophrenia research. Br J Psychiatry 2007; 191 (suppl 50): s42-5.

35 Awad AG, Voruganti LN. The burden of schizophrenia on caregivers: a review. Pharmacoeconomics 2008; 26: 149-62.

We know that antidepressants: get into the brain; need certain pharmacology properties; have acute and chronic neurochemical effects; influence neuronal circuitry underpinning mood; alter emotional processing; have biological actions comprising only a modest part of the overall therapeutic effect. We don't know: the specific pharmacological, neuronal, neuropsychological actions necessary or sufficient for efficacy; how these interact with non-specific and psychological factors; how to effectively sequence treatment based on pharmacology; how to predict who will benefit from which, or any, drug. Effective prescribing remains an art in which how treatment is carried out is as important as what drug is used. 